\title{
Comparative Safety and Efficacy of Treatments for Overactive Bladder Among Older Adults: A Network Meta-analysis
}

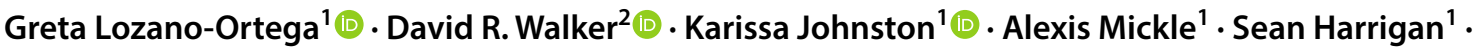 \\ Basia Rogula'® ${ }^{1} \cdot$ Rita M. Kristy ${ }^{2} \cdot$ John C. Hairston ${ }^{2} \cdot$ Carol R. Schermer $^{2}$
}

Published online: 22 September 2020

(c) The Author(s) 2020

\begin{abstract}
Background Cumulative exposure to one or more anticholinergic medications ("anticholinergic burden") is associated with an increased risk of adverse outcomes, particularly among older individuals. Mirabegron, an oral selective $\beta 3$-adrenergic receptor agonist, has demonstrated efficacy in managing the symptoms of overactive bladder without contributing to anticholinergic burden. However, it is not known whether the favorable safety profile of mirabegron relative to antimuscarinics varies with increasing age among a patient population who may have a high anticholinergic burden.

Objective The primary objective of this study was to indirectly compare the safety and efficacy profile of mirabegron relative to antimuscarinics in older adults with overactive bladder.

Methods A systematic literature review was conducted to identify randomized controlled trials that reported safety and efficacy endpoints among patients aged $\geq 65$ years. Identified randomized controlled trials were subsequently synthesized via a network meta-analysis. Preferred Reporting Items for Systematic Reviews and Meta-Analysis guidelines in designing, performing, and reporting the literature review were followed. In line with current best practices, the network meta-analysis was conducted using a Bayesian approach and according to the overall general guidance for evidence synthesis developed by the National Institute for Health and Care Excellence decision support unit. Estimates of relative safety were assessed via the odds ratio and estimates of relative efficacy were assessed via means and credible intervals.

Results A total of 3078 abstracts, 300 of which underwent full-text screening, were identified using the search criteria. Twenty articles reporting on 21 randomized controlled trials were eligible for data extraction and synthesis. Following review, five safety and five efficacy endpoints were considered for inclusion in the network meta-analysis. Regarding findings typical of anticholinergic exposure in older adults, mirabegron was not associated with an increased odds of dry mouth (odds ratio 95\% credible interval 0.76 [0.26-2.37]) or constipation (1.08 [0.39-3.02]) relative to placebo, whereas antimuscarinics were strongly associated with these events (odds ratio range 3.78-7.85 and 2.12-4.66, respectively). In this older population, mirabegron was associated with a similar odds of experiencing adverse event-related treatment discontinuations relative to placebo (0.99 [0.57-1.70]), while the odds of experiencing an adverse event-related treatment discontinuation for antimuscarinics had a range of 1.14-3.03 (in most cases, the association was mild). No increased odds of experiencing overall treatment-emergent adverse events was observed for mirabegron or antimuscarinics (odds ratio range 1.25-1.55), apart from fesoterodine (2.23 [1.37-3.37]). Finally, a similar treatment effect was observed across all efficacy endpoints between mirabegron and antimuscarinics in this older population.

Conclusions This study indicates that the safety and efficacy profile of mirabegron remains favorable compared with antimuscarinics among older adults. This includes safety outcomes typically associated with anticholinergic burden, which were less frequently observed in patients treated with mirabegron.
\end{abstract}

Extended author information available on the last page of the article 


\section{Key Points}

This study assesses the comparative safety and efficacy of antimuscarinics and mirabegron among older adults (aged $\geq 65$ years) with overactive bladder at risk of a high anticholinergic burden via a synthesis of available evidence.

Results suggest that the favorable safety profile of mirabegron is maintained among older adults with overactive bladder compared to antimuscarinics.

Mirabegron is similarly efficacious to antimuscarinics when used in this population and does not contribute to adverse events typically associated with anticholinergic burden.

\section{Introduction}

Anticholinergic medications are commonly prescribed among older adults and are indicated for a range of conditions, including respiratory disease (asthma, chronic obstructive pulmonary disease), neurological/psychiatric conditions (depression, psychosis, Parkinson's disease), gastrointestinal disorders, allergies, and overactive bladder (OAB) [1-3]. As many of these conditions increase in prevalence with age, it is unsurprising that up to half of older adults have been reported to have exposure to anticholinergic medications, sometimes multiple agents simultaneously [4]. Additionally, cumulative exposure to anticholinergic medications, termed "anticholinergic burden," is directly related to an increased risk of adverse effects (AEs) and their associated outcomes [5]. Among these are increased morbidity, which lead to a greater risk of costly outcomes such as hospitalization and institutionalization, and a greater risk of mortality $[3,6]$.

Anticholinergic medications have been associated with a variety of unwanted effects, including dry mouth, constipation, falls, and fractures, as well as neurological and behavioral issues such as delirium, cognitive impairment, and confusion $[1,3,5,6]$. Most recently, studies have found that exposure to certain anticholinergic medications, including some anti-depressants, bladder antimuscarinics, anti-epileptic drugs, and anti-Parkinson's disease drugs, is associated with an increased risk of dementia, which is particularly concerning in an older population $[7,8]$. This has resulted in increased attention to the high anticholinergic burden among older adults and recognition of the need to reduce exposure to these medications.

For OAB treatment, antimuscarinics, which belong to the anticholinergic class of medications, are commonly used as first-line pharmacotherapy [9]. However, options from other classes of medications are available; alternative pharmacologic interventions in $\mathrm{OAB}$ management that do not contribute to anticholinergic burden include mirabegron, an oral $\beta 3$-adrenergic receptor agonist that has demonstrated similar efficacy to antimuscarinics, but with a more favorable safety profile [10].

The degree to which the favorable safety profile of mirabegron relative to antimuscarinics is affected by high levels of anticholinergic burden is still poorly understood, although recent literature has highlighted the relationship between adverse outcomes and anticholinergic burden in OAB [11-13]. A comprehensive assessment is precluded by the lack of literature from randomized controlled trials (RCTs) conducted in individuals with high anticholinergic burden; however, older adults can act as a proxy given their commonly high exposure to these types of medications. By examining the comparative safety and efficacy of antimuscarinics and mirabegron among older adults with $\mathrm{OAB}$, a better understanding of treatment outcomes in a population with widespread use of anticholinergics can be generated. Therefore, the primary objective of this study was to estimate the safety and efficacy profile of mirabegron relative to antimuscarinics in older adults with $\mathrm{OAB}$.

\section{Methods}

This study consisted of two parts, including a systematic literature review (SLR) and a subsequent network metaanalysis (NMA) of published evidence from RCTs. Safety and efficacy parameters identified within the SLR were synthesized using NMA methodology to assess the study objectives.

\subsection{Systematic Literature Review}

The purpose of the SLR was to identify all published RCTs conducted among older adults with OAB that reported the safety and efficacy outcomes associated with the use of mirabegron and/or antimuscarinics. As such, the design and implementation of the systematic review was guided by the PICOS (Population, Interventions/Comparators, Outcomes, Study design) criteria to maximally enable the identification of all applicable studies (Table 2) [14, 15]. Studies that reported $\mathrm{OAB}$ treatments (solifenacin, tolterodine, darifenacin, fesoterodine, oxybutynin, trospium, mirabegron) and doses that are approved in the USA were eligible for inclusion in the review, regardless of the countries in which the trials were conducted. Furthermore, to ensure that outcomes were applicable to the population of interest, only studies where at least $80 \%$ of participants were aged 65 years or older, or those that reported results separately for this subgroup of individuals, were considered. This $80 \%$ threshold 
was defined a priori to ensure that the majority of the evidence applied to the target population.

Studies were excluded if participants had a known etiology of their bladder dysfunction such as neurogenic detrusor overactivity, stress urinary incontinence, bladder oversensitivity, bladder hypersensitivity, nocturia only, or interstitial cystitis only. Additionally, phase I studies and cross-over studies where results were not reported before cross-over occurred were also excluded. Studies were not restricted by a patient's prior anticholinergic use; however, a washout period of several weeks was often implemented, and concomitant use during the trials was either restricted or not reported.

The SLR was conducted using MEDLINE, EMBASE, and PubMed. The search was limited to English language publications including recent conference abstracts from 1 January, 2000 to 21 August, 2018; the detailed search strategy is presented in Appendix 1. Studies were evaluated for inclusion via a two-phased process. First, two researchers worked independently to review all titles and abstracts identified by the search strategy against the PICOS criteria [15]. Next, a full-text double-parallel review was conducted for studies where a decision regarding study inclusion was not possible based on the information contained in the title and abstract. If any discrepancies occurred between the studies selected for inclusion by the two researchers, a third researcher provided arbitration. At this point, studies where overlapping populations were evident were identified and those with the most complete data were kept.

All stages of the SLR were conducted using the tracking system DistillerSR ${ }^{\circledR}$. Preferred Reporting Items for Systematic Reviews and Meta-Analysis (PRISMA) guidelines in designing, performing, and reporting the literature review were followed [16]. Following completion of the SLR, data were extracted by a researcher into a customized Microsoft ${ }^{\circledR}$ Excel $^{\circledR}$ workbook for all study design, demographic, clinical, and outcomes data of interest from the included studies. While not reported within all studies, details of anticholinergic burden were extracted when available using the scales and measures as reported in the original publications. A second researcher reviewed the extraction for accuracy.

\subsection{Network Meta-Analysis}

\subsubsection{Overview}

Following the extraction of data from studies included in the SLR, an NMA was conducted to synthesize the identified safety and efficacy parameters associated with mirabegron and antimuscarinic use. Following completion of the SLR, a feasibility assessment was carried out and inclusion was limited to studies with endpoints reported at 12 weeks ( \pm 1 week) in the NMA to ensure a homogeneous evidence base. To ensure transparency and alignment with best practices, the NMA was conducted using a Bayesian approach and in accordance with the general guidance for evidence synthesis developed by the National Institute of Health and Care Excellence Decision Support Unit [15, 16].

\subsubsection{Endpoint Selection}

Endpoints were selected for inclusion into the NMA following a feasibility assessment of the data obtained from the SLR. Regarding safety endpoints, AEs known to be related to the intervention and comparators of interest were pre-specified for consideration. Both safety and efficacy endpoints were included in the NMA when sufficient data existed to formulate a connected network between mirabegron and antimuscarinics, and when there was consistency in the evidence base with respect to trial patient populations and outcome definitions. This was particularly relevant for safety endpoints, given the potential for variability in definitions (e.g., thresholds or markers) used across trials. All endpoints were extracted overall and according to levels of anticholinergic burden when possible. Finally, analyses based on the modified intention-to-treat population (i.e., participants who have had at least one dose of the intervention) were favored over intention to treat (i.e., all participants randomized) or per protocol, if available [17].

All efforts were made to obtain or impute unavailable data elements to maximize the evidence available for the analysis. For continuous endpoints, whenever changes from baseline were not reported, change was subsequently derived from baseline and final estimates, if available. If a study only reported medians, these were used to impute the means only when the study was the sole source of evidence for an individual comparator. For binary endpoints, whenever the proportion was reported, but not the frequency, the latter was estimated based on the number of patients included in each arm and the reported proportion. These data were checked against estimates reported in the literature for the full study sample to ensure consistency of estimates. For studies that reported endpoints for different age subgroups (e.g., 65-74 years, and 75 years and older), results were pooled at the treatment level in preparation for analysis. Last, imputation of missing standard deviations (SDs) were conducted according to recommendations from the Cochrane Handbook for Systematic Reviews of Interventions [18].

\subsection{Statistical Analyses}

All analyses were conducted using $\mathrm{R}$ version 3.6.0 and package R2WinBUGS version 2.121[19]. The NMA was 
conducted using both fixed-effect (FE) and random-effect (RE) models, with goodness of fit guided by the deviance information criterion (DIC). Vague prior distributions were used to estimate the between-study variance. For each endpoint, the RE model was selected a priori, unless either the FE provided a considerably better fit based on the DIC (at least a 3-point difference) or the between-study variance estimated by the RE model was considered non-informative (i.e., the posterior credible interval [CrI] for the between study variance was too wide).

In the base-case analysis, mirabegron and individual antimuscarinics were compared to placebo (reference treatment). The base-case analysis was conducted assuming, a priori, homogeneity in the evidence base. The validity of this assumption was evaluated via estimation of the betweenstudy SD within the RE model. For each endpoint, if there was evidence of heterogeneity in the evidence base, the following baseline characteristics were considered for covariate adjustment in the NMA via meta-regression: baseline disease severity, study duration (or time at which endpoint was reported), type of OAB (incontinence vs urgency vs mixed), time since diagnosis, age at study entry, proportion who were treatment naïve, and sex. Additionally, pairwise meta-analyses were performed for all direct comparisons informed by two or more studies, under both FE and RE approaches, to further explore the presence of heterogeneity in the evidence base, beyond endpoint definition.

Continuous outcome variables were modeled using a normal likelihood function with an identity link, while binary outcome variables were modeled using a binomial likelihood with a logit link. Estimates of relative safety were assessed via the odds ratio (OR) and estimates of relative efficacy were assessed via means and CrI. Heterogeneity between studies was addressed by comparing networks with studies included or excluded.

Sensitivity analyses were conducted for endpoints where the RE model did not accurately predict the between-study SD. Antimuscarinics were pooled into a common comparator for these analyses, which led to a simplification of the network structure and a considerable reduction in the number of parameters being estimated. This was only feasible when there was evidence of similar efficacy and/or safety across antimuscarinics (i.e., homogeneity of the evidence across antimuscarinics).

Given the Bayesian approach of these analyses (which precludes use of frequentist statistical significance measures), the magnitude of the benefit was described based on the proportion of the CrI that fell on the point estimate side of equivalence: weak if the percentage of the $\mathrm{CrI}$ was $<90 \%$, moderate if the percentage of the CrI was $\geq 90 \%$, and strong if the CrI was entirely on one side of equivalence.

\section{Results}

\subsection{Systematic Literature Review Results}

A total of 3078 abstracts, 300 of which underwent full-text screening, were identified using the search criteria. After the exclusion of studies with overlapping populations that provided no additional data [20-27], 20 articles reporting on 21 RCTs were eligible for data extraction and synthesis [23, 28-46]. Following the feasibility assessment, 14 were included in the NMA [23, 28-32, 36, 38-41, 43-45] [Fig. 1].

Key characteristics of the included studies are reported in Table 1. All therapies eligible for inclusion based on PICOS criteria were captured in the identified studies. Among the 21 double-blind studies identified in this systematic review, there were ten pooled analyses $[29,31,32,34,36,39,40$, 42, 43], nine phase III studies [28, 29, 32, 39, 40, 42, 43, 46], and one phase IV study [44]; 11 others did not report the study phase but were generally consistent with features of phase III/IV studies, based on study design and sample size $[23,30,31,33-38,41,45]$. Total sample size of patients aged $\geq 65$ years had a range from 72 in Frenkl et al. [33] to 1632 in Griebling et al. [34].

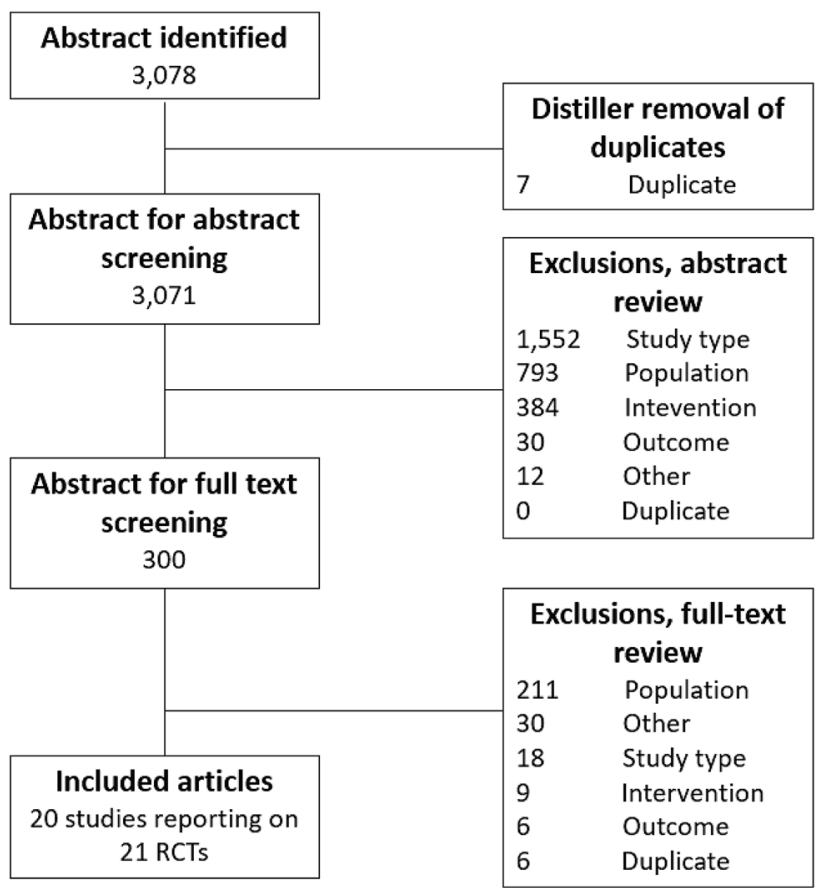

Fig. 1 Preferred Reporting Items for Systematic Reviews and MetaAnalysis (PRISMA) diagram of publication flow through the systematic literature review. RCTs randomized controlled trials 


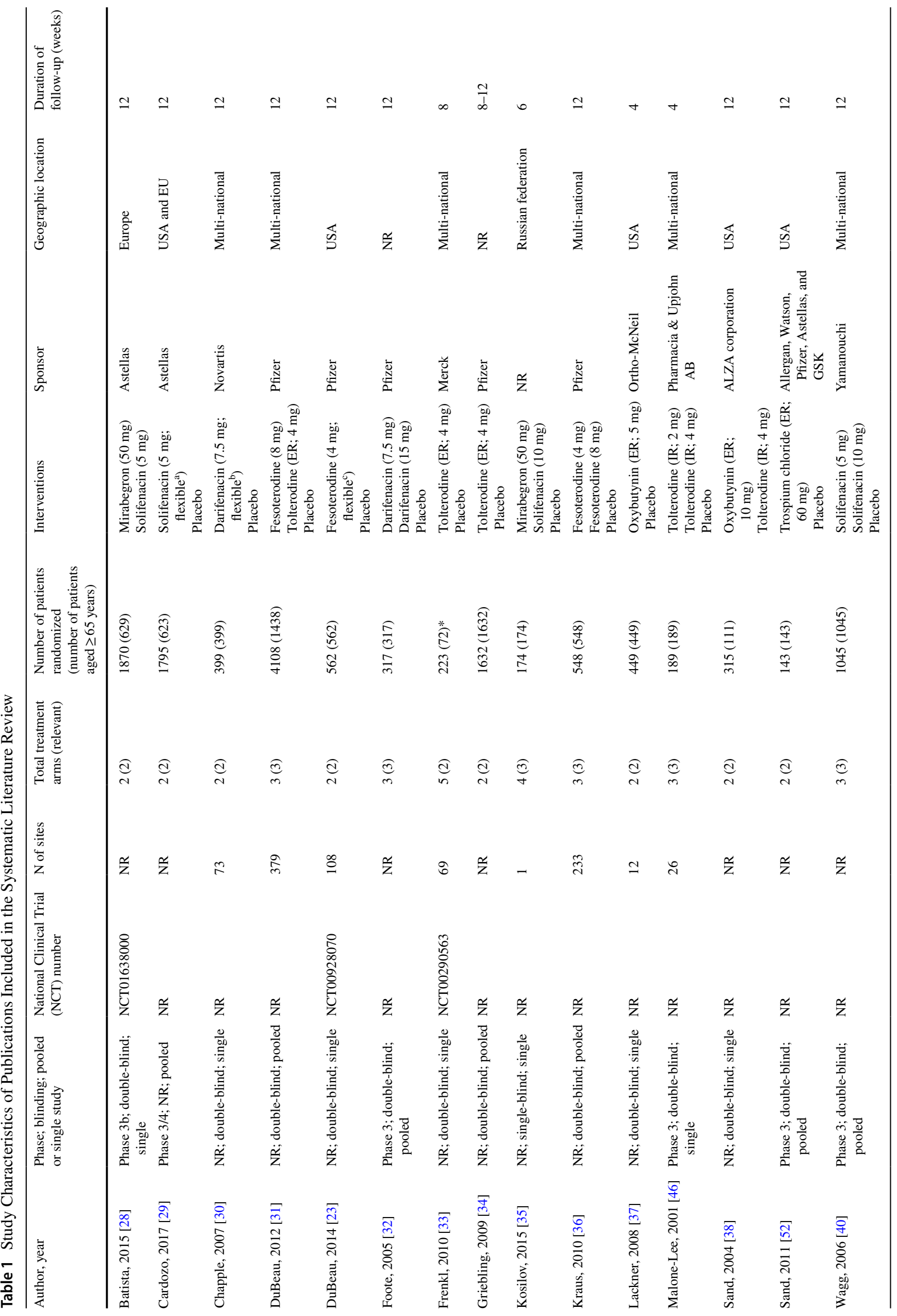




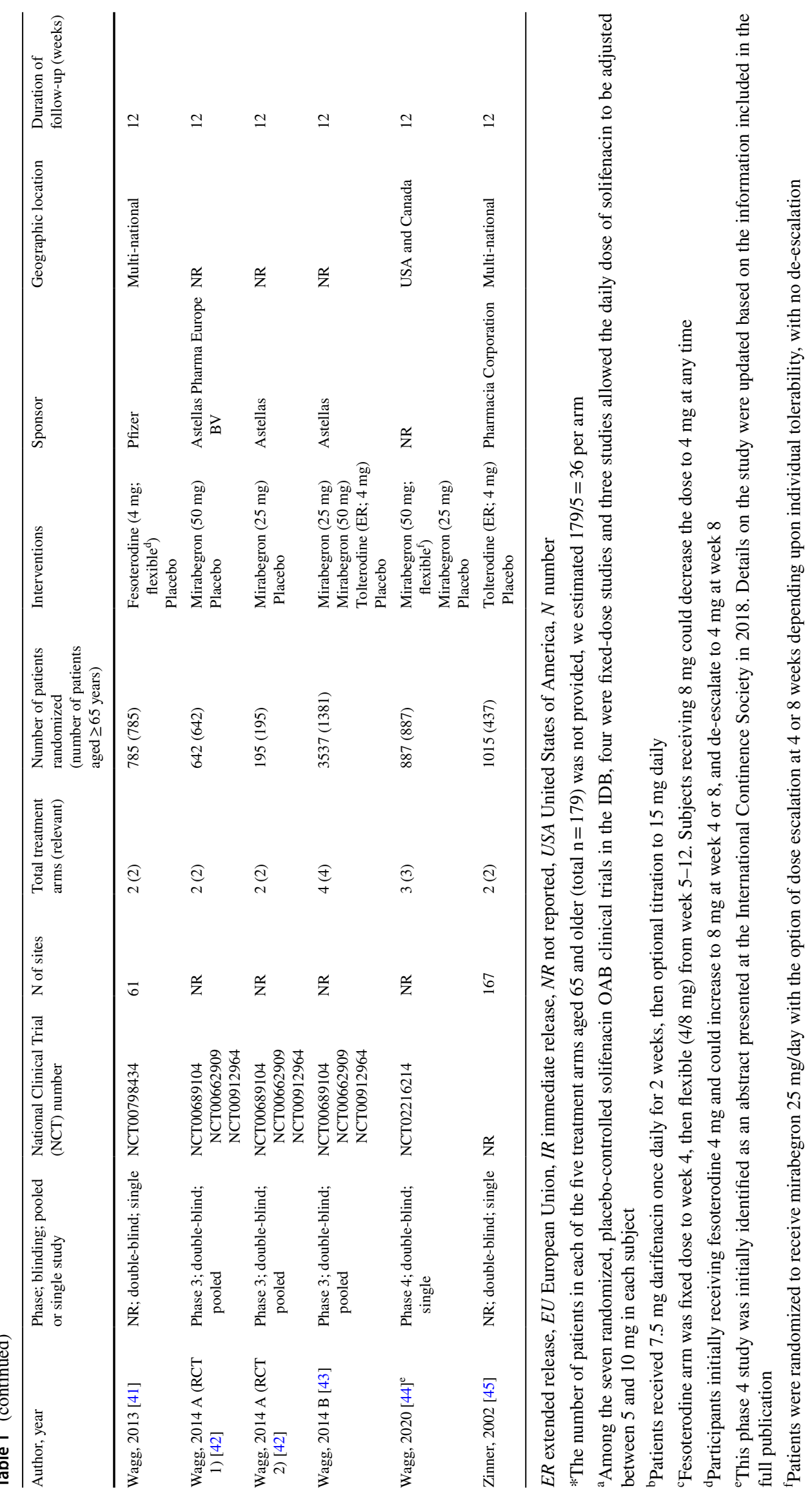




\subsection{Study Endpoints}

Following the feasibility assessment, ten endpoints were considered for inclusion in the NMA. These included five safety endpoints (urinary retention, dry mouth, constipation, overall treatment-emergent adverse events [TEAEs], and AE-related treatment discontinuations), and five efficacy endpoints (incontinence episodes per $24 \mathrm{~h}$, urgency incontinence episodes per $24 \mathrm{~h}$, micturitions per $24 \mathrm{~h}$, volume voided per micturition, and urgency episodes per $24 \mathrm{~h}$ ). The overall network diagram, as well as the corresponding diagrams for each safety and efficacy endpoint, is presented in Fig. 2.

\subsection{Safety Results}

Twelve studies contributed to the safety results $[23,29-32$, $36,38,39,41,43-45]$, including 11 studies and 7170 patients contributing evidence on the incidence of dry mouth and constipation [23, 29-32, 36, 38, 39, 41, 43, 45], seven studies and 6374 patients on overall TEAEs [29-31, 41, 43-45], and eight studies and 6937 patients on AE-related treatment discontinuations [23, 29-31, 41, 43-45]. Some studies only presented overall TEAEs among the entire study population rather than only among those aged 65 years or older [38], or rates of specific TEAEs were reported individually and not overall $[32,36]$. One study reported overall TEAEs for events that occurred more often in the treatment arm vs the placebo arm [23], and one study reported only TEAEs considered related to study medication (i.e., treatment related vs emergent), not overall [39]. Under these circumstances, they could not be included in the analyses. Additionally, while urinary retention was initially considered for inclusion following the feasibility assessment, a low event count across trials and the subsequent failure of models to reach convergence precluded evidence synthesis via the NMA.

For each of the four remaining safety endpoints, RE models were chosen over FE models to estimate the ORs. While DIC statistics indicated that the FE models provided a modestly better fit than the RE models for constipation (RE DIC: 145.4 vs FE DIC: 143.4), TEAEs (RE DIC: 125.7 vs FE DIC: 124.4), and AE-related treatment discontinuations (RE DIC: 112.4 vs FE DIC: 110.5$)$, the RE models were nonetheless favored because of the difference in DIC not exceeding the prespecified threshold as well as their ability to estimate

Fig. 2 Network diagram: overall and by safety and efficacy endpoint. $A E$ adverse event, $D A R$ darifenacin, FES festerodine, $M I R$ mirabegron, $O X Y$ oxybutynin, $P L$ placebo, $S O L$ solifenacin, TEAEs treatmentemergent adverse events, $T O L$ tolterodine, TRO trospium chloride

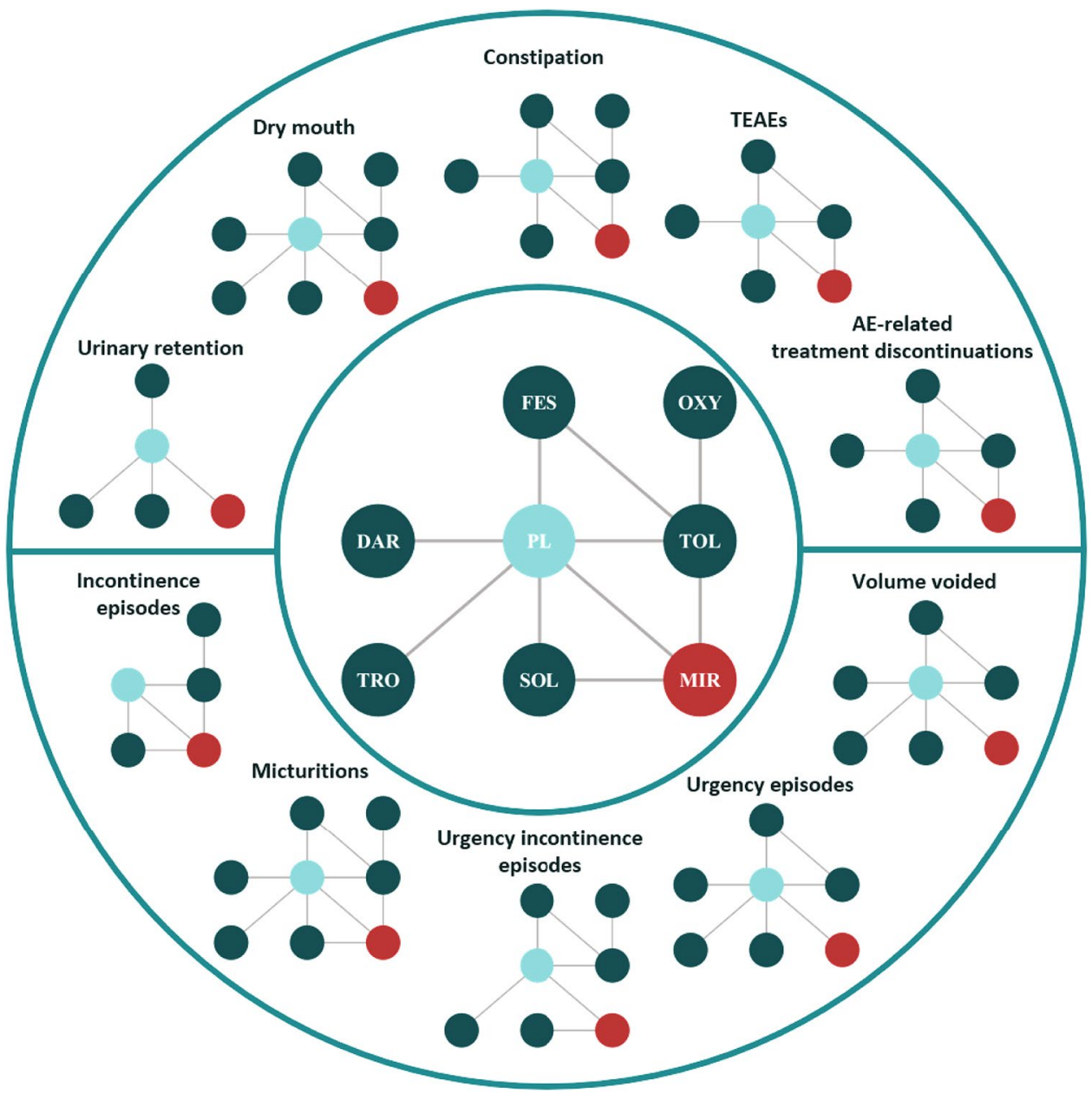


the between-study SD (SD, 95\% CrI 0.2 [0.0-0.8] for constipation, 0.2 [0.0-0.7] for overall TEAEs, and 0.2 [0.0-0.7] for AE-related treatment discontinuation). For dry mouth, the RE model was associated with the best fit (RE DIC: 163.4 vs FE DIC: 164.6 ; SD, 95\% CrI 0.4 [0.0-1.0]). Results from the non-selected fixed effect model are provided in Appendix 2.

The TEAE endpoint met the criteria for sensitivity analyses, where all antimuscarinics were pooled into a common comparator, reducing the network to a three-node network. In this case, the RE model provided the best fit to the data and more precisely estimated the between-study SD $(0.3$ [0.2-0.7]).

Results from the RE models are presented in Fig. 3. For both dry mouth and constipation, mirabegron was not associated with an increased odds of these events relative to placebo (OR, 95\% CrI 0.76 [0.26-2.37] and 1.08 [0.39-3.02], respectively). Conversely, antimuscarinics were strongly associated with an increased odds of dry mouth and constipation. Trospium was excluded from the final results for constipation, given that no events were reported for the placebo group, which led to parameter instability.

Neither mirabegron nor antimuscarinics were strongly associated with an increased odds of overall TEAEs, with the exception of fesoterodine, in the base case. In the sensitivity analysis, antimuscarinics were strongly associated with an increased odds for TEAEs relative to placebo (1.46 [1.05-2.05]), while mirabegron was weakly associated with a higher odds relative to placebo (OR, 95\% CrI 1.32 [0.78-2.27]).

Finally, mirabegron was not associated with an increased risk of AE-related treatment discontinuations relative to placebo. Fesoterodine was strongly associated with an increased odds of experiencing AE-related treatment discontinuations relative to placebo, whereas the association among all other antimuscarinics was weak.

\subsection{Efficacy Results}

Thirteen studies contributed to the efficacy results $[23,28$, 30-32, 36, 38-41, 43-45], including six studies and 3317 patients contributing evidence on incontinence episodes per $24 \mathrm{~h}[28,38,40,42,44,45]$, eight studies and 4878 patients on urgency incontinence episodes per $24 \mathrm{~h}[23,28,31,36$, $38,39,41,44], 13$ studies and 8313 patients on micturitions per $24 \mathrm{~h}[23,28,30-32,36,38-42,44,45]$, seven studies and 4610 patients on volume voided per micturition [31, 32, $36,39,40,44,45]$, and nine studies and 5847 patients on urgency episodes per $24 \mathrm{~h}[23,30-32,36,39-41,44]$.

For both the number of incontinence and urgency incontinence episodes per $24 \mathrm{~h}, \mathrm{RE}$ models were chosen over FE models to estimate the treatment effects. While DIC statistics indicated that the FE models provided a modestly better fit than the RE models in both instances (incontinence RE DIC: 5.1 vs FE DIC 3.6; urgency incontinence RE DIC: 3.6 vs FE DIC: 1.8), the RE models were nonetheless favored owing to the difference in DIC not exceeding the prespecified threshold/as well as their ability to estimate the between-study SD (SD, 95\% CrI 0.3 [0.0-1.3] for incontinence, 0.2 [0.0-1.1] for urgency incontinence).

For micturitions per $24 \mathrm{~h}$, the RE model was associated with the best fit (RE DIC: 7.8 vs FE DIC: 18.2 ; SD, 95\% CrI $0.4[0.1,0.8])$ and therefore chosen over the FE model. For both volume voided per micturition and number of urgency episodes per $24 \mathrm{~h}$, FE models were chosen over RE models. The DIC statistic favored the RE models for both endpoints (volume voided per micturition RE DIC: 92.1 vs FE DIC 116.1; urgency episodes per 24 h RE DIC: 11.3 vs FE DIC: 33.8). However, FE models were chosen in both instances because of the inability of the RE models to accurately estimate the between-study SD (28.8 [8.3-82.1] for volume voided per micturition; 1.3 [0.5-3.7] for urgency episodes per $24 \mathrm{~h}$ ) with respect to the uninformative prior distributions (volume voided per micturition: uniform 0-300; urgency episodes per $24 \mathrm{~h}$ : uniform 0-100).

Results for the models are presented in Fig. 4. Among all endpoints, the degree of treatment effect associated with mirabegron and antimuscarinics was variable. Compared to antimuscarinics, mirabegron had the strongest association with the reduction in incontinence episodes per $24 \mathrm{~h}$, relative to placebo (mean $95 \% \mathrm{CrI}-0.65$ episodes [ -1.23 to $-0.10]$. While solifenacin was associated with a slightly higher reduction in incontinence episodes, the inclusion of the null value in the $95 \% \mathrm{CrI}$ indicated the presence of a weaker evidence base ( -0.67 episodes [ -1.39 to 0.01$]$ ). Similarly, fesoterodine had the strongest evidence for its association with a reduction in urgency incontinence episodes ( -0.43 episodes $[-0.81$ to -0.05$])$, while mirabegron and solifenacin were each associated with a larger reduction but weaker evidence (mirabegron: -0.57 episodes [ -1.31 to 0.17$]$; solifenacin: -0.61 episodes [ -1.74 to $0.52]$ ). For micturitions, volume voided per micturition, and number of urgency episodes, strong associations among both mirabegron and antimuscarinics (with the exception of oxybutynin and tolterodine) were observed.

Finally, a sensitivity analysis was conducted for a number of urgency episodes per $24 \mathrm{~h}$ owing to the overlapping CrIs across antimuscarinics. Subsequently, all antimuscarinics were pooled into a common comparator, reducing the network to a three-node network. In this case, the RE model was selected over the FE model (between-study SD: 0.8 [0.4-1.6]). Both antimuscarinics and mirabegron were associated with a clinical benefit; however, the evidence was strong only for antimuscarinics (mirabegron: -0.93 episodes [ -2.79 to 0.93$]$; antimuscarinics: -1.04 episodes [ -1.70 to -0.38$]$ ) [Appendix 2]. 
Fig. 3 Relative treatment effects on safety endpoints (randomeffects models). Bold indicates the beta-3 adrenergic agonist class. $C r I$ credible interval, $D A R$ darifenacin, FES festerodine, $M I R$ mirabegron, $O R$ odds ratio, $O X Y$ oxybutynin, $P L$ placebo, $S A$ sensitivity analysis, $S O L$ solifenacin, TOL tolterodine, $T R O$ trospium chloride

\begin{tabular}{|c|c|}
\hline Intervention & OR $(95 \% \mathrm{Crl})$ \\
\hline \multicolumn{2}{|l|}{ Dry mouth } \\
\hline Mirabegron (MIR) & $0.76(0.26,2.37)$ \\
\hline Placebo (PL) & reference \\
\hline Tolterodine (TOL) & $3.78(2.17,7.43)$ \\
\hline Trospium chloride (TRO) & $4.10(0.68,33.08)$ \\
\hline Oxybutynin (OXY) & $4.62(1.25,18.73)$ \\
\hline Fesoterodine (FES) & $7.17(4.18,11.86)$ \\
\hline Solifenacin (SOL) & $7.24(2.57,20.95)$ \\
\hline Darifenacin (DAR) & $7.85(3.17,21.22)$ \\
\hline
\end{tabular}

Constipation Placebo (PL) Mirabegron (MIR) Tolterodine (TOL) Darifenacin (DAR) Fesoterodine (FES) Oxybutynin (OXY)

\section{TEAEs}

Placebo (PL)

Tolterodine (TOL)

Mirabegron (MIR)

Solifenacin (SOL)

Darifenacin (DAR)

Fesoterodine (FES)

$7.85(3.17,21.22)$

reference

$1.08(0.39,3.02)$

$2.12(1.18,3.74)$

$2.83(1.43,5.93)$

$3.16(1.97,5.24)$

$4.31(1.87,10.04)$

$4.66(0.98,23.76)$

reference

$1.25(0.89,1.72)$

$1.26(0.87,1.86)$

$1.45(0.82,2.55)$

$1.55(0.81,2.96)$

$2.23(1.37,3.37)$ Solifenacin (SOL)
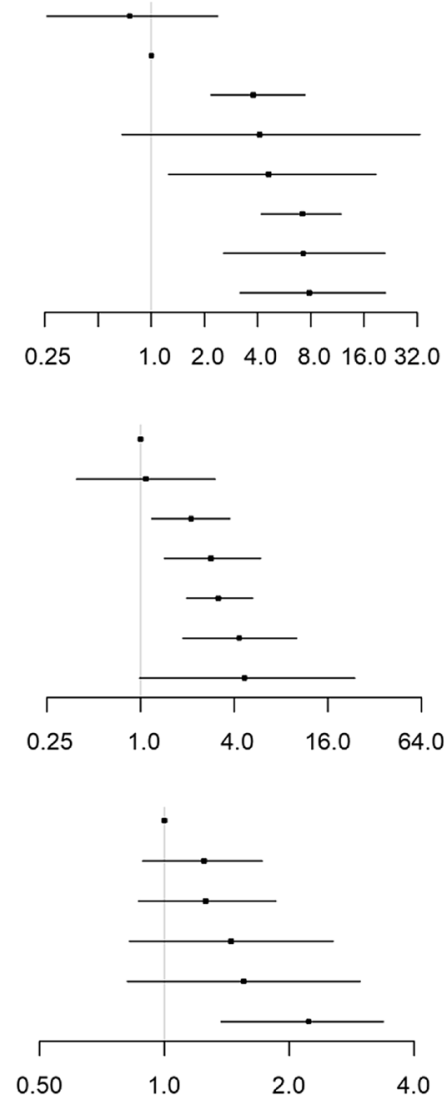

TEAEs: SA with pooled AMs
Placebo (PL)

Mirabegron (MIR)
Antimuscarinic (AM) reference

$1.32(0.78,2.27)$

$1.46(1.05,2.05)$

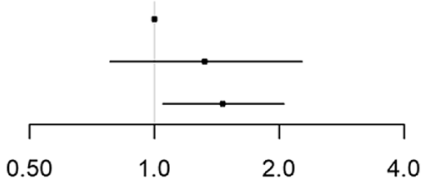

AE-related treatment discontinuations Mirabegron (MIR) Placebo (PL) Tolterodine (TOL) Solifenacin (SOL) Fesoterodine (FES) Darifenacin (DAR)

$$
\begin{array}{r}
\mathbf{0 . 9 9}(\mathbf{0 . 5 7 , ~ 1 . 7 0 )} \\
\text { reference } \\
1.14(0.70,1.81) \\
1.22(0.58,2.54) \\
2.23(1.44,3.48) \\
3.03(0.31,80.48)
\end{array}
$$

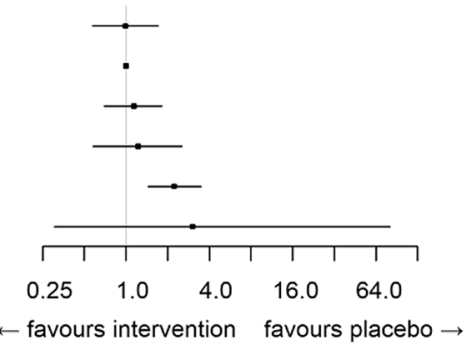

\section{Discussion}

This study characterized the safety and efficacy of mirabegron relative to antimuscarinics in older adults with $\mathrm{OAB}$ via an NMA of published data. To the authors' knowledge, this is the first attempt to synthesize evidence among a patient population with a potentially high use of anticholinergics.

The synthesis of published evidence presented here suggests that antimuscarinics and mirabegron are similarly efficacious among older adults. Regarding adverse events, antimuscarinics were found to be strongly associated with an increased odds of experiencing dry mouth and constipation relative to placebo, whereas mirabegron was not. This is unsurprising given that these adverse events are typically associated with anticholinergic use. Notably, although urinary retention was initially considered for inclusion as an outcome of interest as it has historically been considered a high-risk event among patients with $\mathrm{OAB}$, it was dropped 
Fig. 4 Relative treatment effects on efficacy endpoints (randomeffects models). Bold indicates the beta- 3 adrenergic agonist class. $\mathrm{CrI}$ credible interval, $D A R$ darifenacin, FES festerodine, $M I R$ mirabegron, $O X Y$ oxybutynin, $P L$ placebo, $S O L$ solifenacin, $T O L$ tolterodine, TRO trospium chloride, $*$ fixed affect model

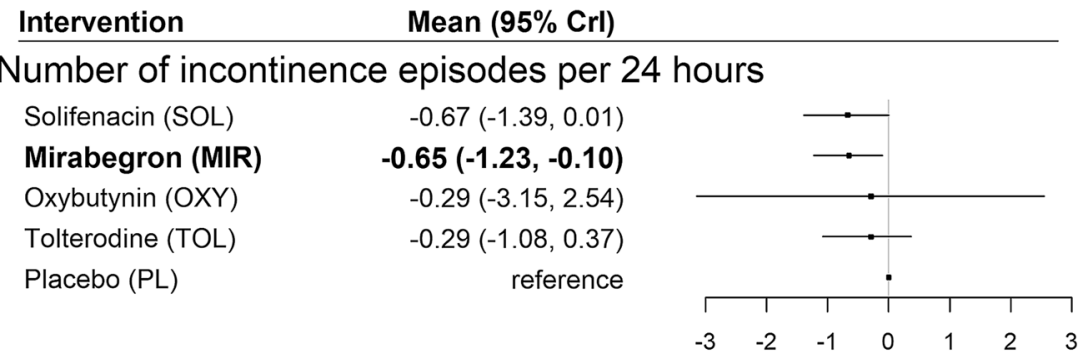

Number of urgency incontinence episodes per 24 hours

$\begin{array}{lr}\text { Trospium chloride (TRO) } & -1.23(-2.51,0.05) \\ \text { Solifenacin (SOL) } & -0.61(-1.74,0.52) \\ \text { Mirabegron (MIR) } & \mathbf{- 0 . 5 7 ( - 1 . 3 1 , 0 . 1 7 )} \\ \text { Fesoterodine (FES) } & -0.43(-0.81,-0.05) \\ \text { Oxybutynin (OXY) } & -0.37(-2.01,1.25) \\ \text { Tolterodine (TOL) } & -0.20(-0.86,0.45) \\ \text { Placebo (PL) } & \text { reference }\end{array}$

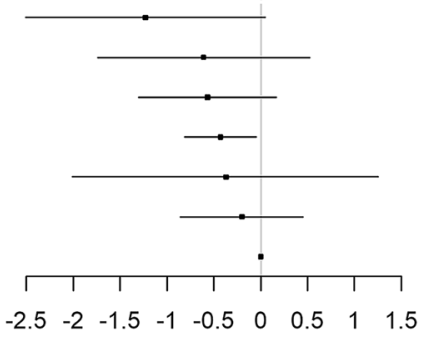

Total number of micturitions per 24 hours

$\begin{array}{lr}\text { Trospium chloride (TRO) } & -1.78(-3.00,-0.56) \\ \text { Solifenacin (SOL) } & -1.12(-1.85,-0.39) \\ \text { Darifenacin (DAR) } & -0.80(-1.54,-0.06) \\ \text { Mirabegron (MIR) } & \mathbf{- 0 . 7 2 ( - 1 . 2 9 , - 0 . 1 4 )} \\ \text { Fesoterodine (FES) } & -0.60(-1.06,-0.12) \\ \text { Oxybutynin (OXY) } & -0.58(-2.31,1.16) \\ \text { Tolterodine (TOL) } & -0.45(-0.98,0.07) \\ \text { Placebo (PL) } & \text { reference }\end{array}$

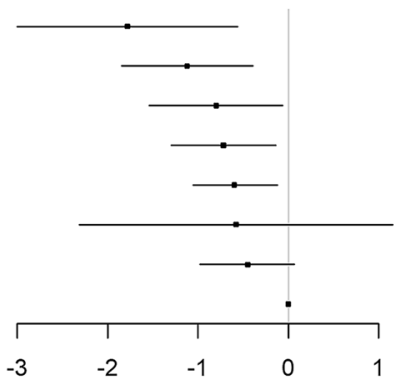

Number of urgency episodes per 24 hours*

$\begin{array}{lr}\text { Trospium chloride (TRO) } & -1.92(-3.07,-0.76) \\ \text { Solifenacin (SOL) } & -1.60(-2.06,-1.13) \\ \text { Fesoterodine (FES) } & -1.04(-1.29,-0.78) \\ \text { Mirabegron (MIR) } & -0.93(-1.48,-0.39) \\ \text { Darifenacin (DAR) } & -0.90(-1.46,-0.35) \\ \text { Tolterodine (TOL) } & -0.48(-0.88,-0.08) \\ \text { Placebo (PL) } & \text { reference }\end{array}$

$\begin{array}{lr}\text { Volume voided per micturition* } \\ \text { Solifenacin (SOL) } & 32.07(25.96,38.22) \\ \text { Trospium chloride (TRO) } & 27.43(12.91,41.98) \\ \text { Darifenacin (DAR) } & 18.90(9.03,28.85) \\ \text { Mirabegron (MIR) } & \mathbf{1 2 . 5 5 ( 3 . 5 9 , 2 1 . 4 6 )} \\ \text { Fesoterodine (FES) } & 12.18(6.60,17.76) \\ \text { Tolterodine (TOL) } & 9.86(4.52,15.20) \\ \text { Placebo (PL) } & \text { reference }\end{array}$

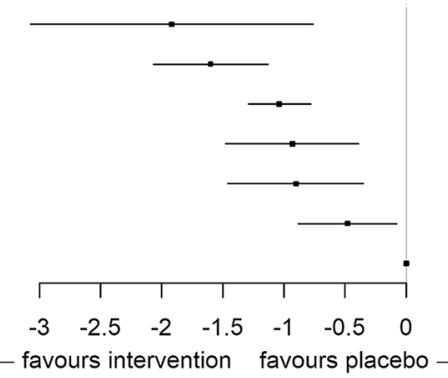

because of a low event count across trials, highlighting that this event may occur with lower frequency than is commonly believed. Overall, these findings are in line with previously published NMAs, where mirabegron was found to be significantly more efficacious than placebo and similarly efficacious to antimuscarinics, but with a statistically and clinically favorable safety profile [47-50]. It should also be noted that previous NMAs of patients with $\mathrm{OAB}$ from all age 
groups have generally indicated a significant improvement on efficacy outcomes associated with solifenacin relative to mirabegron. However, in the present study, there was no strong evidence of this association when the study population was narrowed to older adults.

Because the included studies were primarily RCTs focusing on antimuscarinics, the safety outcomes reported focused on those common to the antimuscarinic class of drugs. As a result, many of the more serious side effects common to beta-3 adrenergic agonists such as hypertension and nasopharyngitis could not be considered in the NMA. Five of the included studies did look at these outcomes. Hypertension/ high blood pressure was reported in $1.1-13.6 \%$ of patients taking mirabegron $[28,35,43]$ vs $1.9-2.4 \%$ of patients taking solifenacin $[28,35] ; 12 \%$ of tolterodine-treated patients [43]; and $6.7-8.4 \%$ of patients receiving placebo [35, 43]. Nasopharyngitis was reported in $0.9-4.5 \%$ of patients taking mirabegron $[43,44]$ vs $3.6 \%$ of patients taking tolterodine and around $2.5 \%$ of patients receiving placebo [43, 44].

Although this study attempted to target a population that is at increased risk for high anticholinergic burden, this concept was not readily ascertainable by the data that comprised the evidence base for the NMA. This was in large part owing to a lack of robust reporting in terms of concomitant medications and highlights an important evidence gap for further research.

\section{Limitations}

There are limitations inherent to this study that warrant mention. Focusing on a population of older adults with OAB led to a reduction in the evidence base compared with that of other NMAs that have compared mirabegron to antimuscarinics. In turn, this reduced the precision of the generated estimates for several endpoints analyzed. Conversely, focusing on older aged individuals may have led to greater homogeneity in the evidence. For instance, any differences in treatment-taking behavior between age groups could result in differences in treatment response.

Furthermore, most of the studies included in the NMA randomized adults of all ages, whereas the results considered here were based on post-hoc analyses on sub-groups who were aged 65 years or older. Therefore, it is unknown whether such results were differentially reported in studies for which positive results were observed in the subgroups, and there is potential for bias in the estimates owing to differences in treatment-effect modifiers that may have been present among those of an older age, given that this subgroup was not specifically a product of randomization. This could not be thoroughly investigated because baseline characteristics for the 65 years of age or older subgroup were not consistently presented across all included studies. However, no major imbalances in patient characteristics were observed for studies that did report baseline characteristics for this age group.

The inclusion of pooled data in the NMA is a further limitation. While it would be preferable to include the individual studies in the NMA, that was not possible as the individual study populations were mixed age groups, and evidence on our target population of older adults was only available in the pooled analyses. It should also be acknowledged that because of the relatively limited pool of evidence, immediate- and extended-release versions of tolterodine and oxybutynin could not be examined separately. However, the majority of identified evidence was for the extended-release versions, as such, the failure to differentiate the immediaterelease studies should have only a minimal impact on the results. Additionally, an assessment of the relative safety of mirabegron and antimuscarinics with regard to urinary retention could not be conducted in this study, as the NMA model was unstable because of very low event counts. It is recognized that alternative methodologies to an evidence synthesis could be considered in these situations, such as the frequentist approach described by Stijnen et al. [51], although the application of alternative methodologies was not performed in this study. Finally, it should be noted that while cognitive effects are among the most worrying potential side effects of pharmaceutical treatments in older adults, unfortunately, the body of evidence provided by clinical trial data for OAB treatments did not permit the exploration of these effects, as this type of information has not been collected to date.

\section{Conclusions}

This study represents an initial exploration into the potential impact that anticholinergic burden may have on the safety and efficacy of $\mathrm{OAB}$ medications, by providing aggregate evidence of the safety and efficacy of mirabegron relative to antimuscarinics among older adults with $\mathrm{OAB}$. The evidence provided by this study indicates that among older adults, the efficacy of mirabegron is similar to that of antimuscarinics. Furthermore, the safety profile of mirabegron relative to that of antimuscarinics remained favorable in this subpopulation of older adults with OAB. This study provides evidence that the safety of antimuscarinics is less favorable relative to mirabegron in this population.

Acknowledgements Medical writing/editorial support was provided by Meagan Harwood MPH from Broadstreet Health Economics and Outcomes Research and funded by the study sponsor.

Author Contributions All authors meet the ICMJE criteria for authorship for this manuscript, take responsibility for the integrity of the work as a whole, and have given final approval to the version to be published. 


\section{Declarations}

Funding The present study was initiated by Astellas Pharma Global Development, Inc., and funding for the conduct of this study was provided by Astellas Pharma Global Development, Inc.

Conflict of interest David Walker, Rita Kristy, John Hairston, and Carol Schermer are employees of Astellas Pharma Global Development, Inc., which sponsored the study. Greta Lozano-Ortega, Karissa Johnston, Basia Rogula, Alexis Mickle, and Sean Harrigan have no conflicts of interest that are directly relevant to the content of this study. Broadstreet Health Economics and Outcomes Research received funding from Astellas Pharma Global Development, Inc. to assist in the development, conduct, and analysis of the study.

Ethics approval Not applicable.

Consent to participate Not applicable.

Consent for Publication Not applicable.

Availability of data and materials Researchers may request access to anonymized participant-level data, trial-level data, and protocols from Astellas-sponsored clinical trials at https://www.clinicalstudydatareq uest.com. For the Astellas criteria on data sharing, see https://www. clinicalstudydatarequest.com/Study-Sponsors/Study-Sponsors-Astel las.aspx.
Code availability Not applicable.

Open Access This article is licensed under a Creative Commons Attribution-NonCommercial 4.0 International License, which permits any non-commercial use, sharing, adaptation, distribution and reproduction in any medium or format, as long as you give appropriate credit to the original author(s) and the source, provide a link to the Creative Commons licence, and indicate if changes were made. The images or other third party material in this article are included in the article's Creative Commons licence, unless indicated otherwise in a credit line to the material. If material is not included in the article's Creative Commons licence and your intended use is not permitted by statutory regulation or exceeds the permitted use, you will need to obtain permission directly from the copyright holder. To view a copy of this licence, visit http://creativecommons.org/licenses/by-nc/4.0/.

\section{Appendix 1}

See Table 2 .

Table 2 Search strategy

\begin{tabular}{|c|c|c|}
\hline \multicolumn{2}{|l|}{ PICOS } & \multirow{2}{*}{$\begin{array}{l}\text { Search term } \\
\text { Urinary bladder, overactive/ or overactive bladder.mp }\end{array}$} \\
\hline Population & 1 & \\
\hline & 2 & Urination Disorders/ \\
\hline & 3 & Exp Lower Urinary Tract Symptoms/ \\
\hline & 4 & Lower Urinary Tract Symptoms.mp \\
\hline & 5 & exp Urinary Incontinence/ or urinary incontinence.mp \\
\hline & 6 & Urinary Bladder Diseases.mp \\
\hline & 7 & exp Enuresis/ or enuresis.mp \\
\hline & 8 & Nocturia.mp \\
\hline & 9 & Urinary Incontinence, Urge/ or urge incontinence.mp \\
\hline & 10 & ((overactive $\$$ or over-activ $\$$ or hyperactive $\$$ or hyper-activ $\$$ or unstable or instability or incontinen $\$$ ) adj3 bladder $\$$ ).tw \\
\hline & 11 & ((urge adj3 incontinence) or (detrusor adj3 dyssynergia) or urinary frequency or bladder irritation or DESD).mp \\
\hline & 12 & $(\mathrm{OAB}$ or $\mathrm{OABS}$ or IOAB or IOABS).tw \\
\hline & 13 & (urge syndrome\$ or urge frequenc\$).tw \\
\hline & 14 & ((overactive $\$$ or over-activ $\$$ or hyperactive $\$$ or hyper-activ $\$$ or unstable or instability) adj3 detrusor $\$) . t w$ \\
\hline & 15 & (urge $\$$ adj3 incontinen\$).tw \\
\hline & 16 & (urin\$ adj3 (incontinen\$ or leak\$ or urgen\$ or frequen\$)).tw \\
\hline & 17 & (urin\$ adj3 (disorder\$ or dysfunct\$)).tw \\
\hline & 18 & (detrusor\$ adj3 (hyperreflexia $\$$ or hyper-reflexia $\$$ or hypertoni $\$$ or hyper-toni\$)).tw \\
\hline & 19 & (micturition\$ adj3 (disorder\$ or dysfunct\$)).tw \\
\hline & 20 & (void\$ adj3 (disorder\$ or dysfunct\$)).tw \\
\hline & 21 & (nocturia or nycturia or enuresis).tw \\
\hline & 22 & or/1-19 \\
\hline
\end{tabular}


Table 2 (continued)

\begin{tabular}{|c|c|c|}
\hline \multicolumn{2}{|l|}{ PICOS } & \multirow{2}{*}{$\begin{array}{l}\text { Search term } \\
\text { exp Muscarinic Antagonists/ or antimuscarinics.mp }\end{array}$} \\
\hline \multirow{10}{*}{$\begin{array}{l}\text { Intervention/ } \\
\text { comparators }\end{array}$} & 23 & \\
\hline & 24 & $\begin{array}{l}\text { (mirabegron or betmiga } \$ \text { or myrbetriq } \$ \text { or betanis } \$ \text { or YM-178 or YM178 or } 223673-61-8 \text { or " } 223673618 \text { " or MVR- } \\
\text { 3JL3B2V).mp }\end{array}$ \\
\hline & 25 & (solifenacin or Vesicare or Vesikur or Vesiker or Vesitirim).mp \\
\hline & 26 & (tolterodine or Detrusitol or Detrol or Detrol LA).mp \\
\hline & 27 & (darifenacin or Enablex or Emselex).mp \\
\hline & 28 & (fesoterodine or Toviaz).mp \\
\hline & 29 & (oxybutynin or Ditropan or Lyrinel XL).mp \\
\hline & 30 & (trospium or Regurin or Flotros or Sanctura or Tropez or Trosec or Spasmex).mp \\
\hline & 31 & Adrenergic beta-3 receptor agonists.mp \\
\hline & 32 & or/23-31 \\
\hline \multirow[t]{27}{*}{ Study type } & 33 & Randomized Controlled Trials as Topic/ \\
\hline & 34 & randomized controlled trial/ \\
\hline & 35 & Random Allocation/ \\
\hline & 36 & Double Blind Method/ \\
\hline & 37 & Single Blind Method/ \\
\hline & 38 & clinical trial/ \\
\hline & 39 & clinical trial, phase ii.pt \\
\hline & 40 & clinical trial, phase iii.pt \\
\hline & 41 & clinical trial, phase iv.pt \\
\hline & 42 & controlled clinical trial.pt \\
\hline & 43 & randomized controlled trial.pt \\
\hline & 44 & multicenter study.pt \\
\hline & 45 & clinical trial.pt \\
\hline & 46 & crossover clinical trial.mp \\
\hline & 47 & exp Clinical Trials as topic/ \\
\hline & 48 & (clinical adj trial\$).tw \\
\hline & 49 & $((\operatorname{sing} 1 \$$ or doubl $\$$ or treb $\$$ or tripl\$) adj (blind $\$ 3$ or mask $\$ 3)) . t w$ \\
\hline & 50 & PLACEBOS/ \\
\hline & 51 & placebo\$.tw \\
\hline & 52 & randomly allocated.tw \\
\hline & 53 & (allocated adj2 random $\$$ ).tw \\
\hline & 54 & or/33-53 \\
\hline & 55 & case report.tw \\
\hline & 56 & letter/ \\
\hline & 57 & historical article/ \\
\hline & 58 & or/55-57 \\
\hline & 59 & 54 not 57 \\
\hline \multirow[t]{5}{*}{ Other } & 60 & 22 and 32 and 59 \\
\hline & 61 & limit 60 to animals \\
\hline & 62 & $60 \operatorname{not} 61$ \\
\hline & 63 & limit 62 to $y r=" 2000-C u r r e n t "$ \\
\hline & 64 & remove duplicates from 63 \\
\hline
\end{tabular}

PICOS Population, Interventions/Comparators, Outcomes, Study design 


\section{Appendix 2: Relative treatment effects on efficacy and safety endpoints (non-selected fixed effect models)}
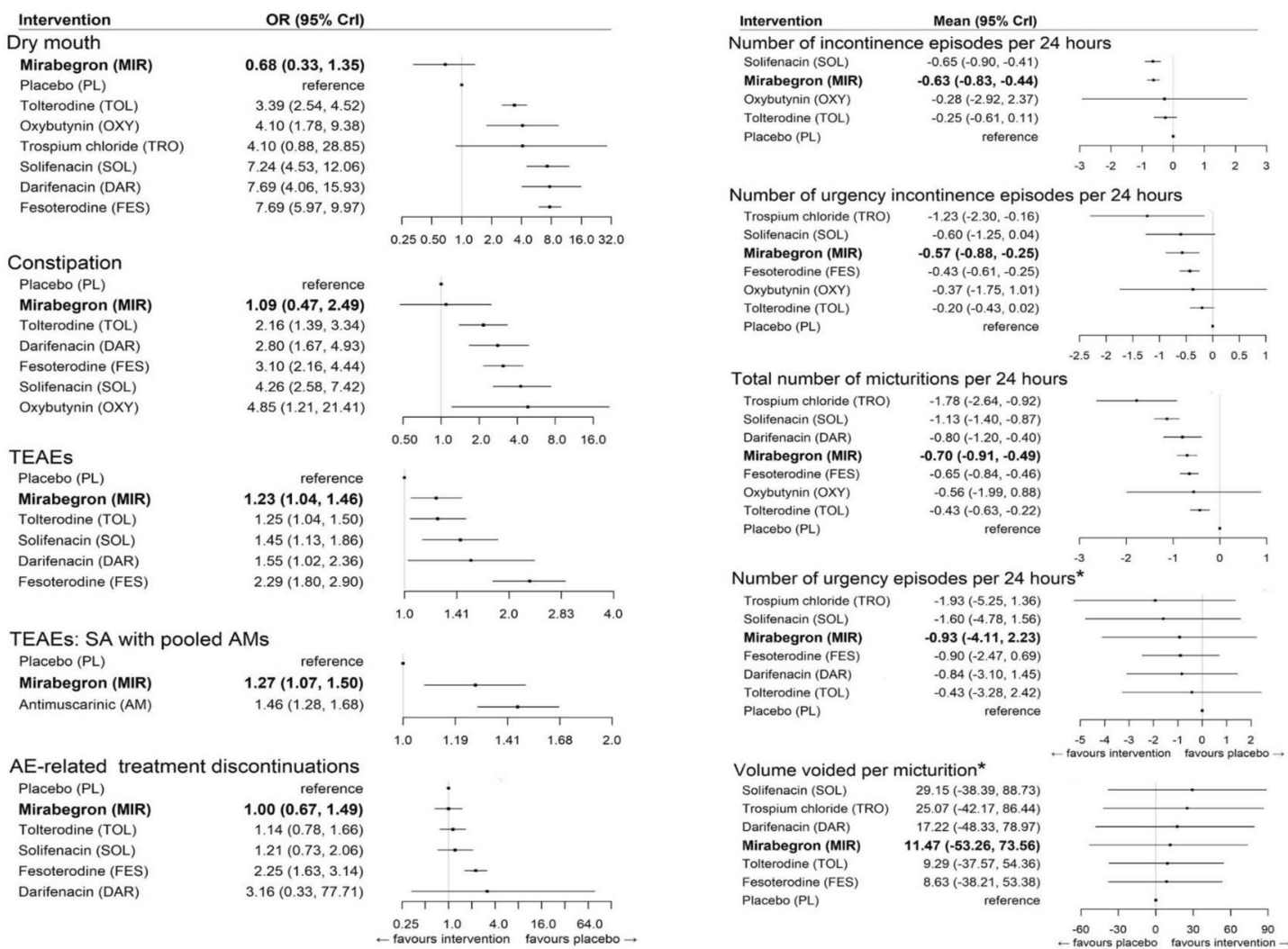

* Random effects model

Abbreviations: $\mathrm{CrI}=$ credible interval; $\mathrm{DAR}=$ darifenacin $\mathrm{FES}=$ festerodine; $\mathrm{MIR}=$ mirabegron; $\mathrm{OR}=$ odds ratio; $\mathrm{OXY}=\mathrm{oxybutynin} ; \mathrm{PL}=$ placebo; $\mathrm{SA}=$ sensitivity analysis; $\mathrm{SOL}=$ solifenacin; $\mathrm{TOL}=$ tolterodine; $\mathrm{TRO}=$ trospium chloride

\section{References}

1. Araklitis G, Cardozo L. Safety issues associated with using medication to treat overactive bladder. Expert Opin Drug Saf. 2017;16(11):1273-80.

2. Geoffrion RN. 283: treatments for overactive bladder: focus on pharmacotherapy. J Obstet Gynaecol Can. 2018;40(1):e22-32.

3. Ruxton K, Woodman RJ, Mangoni AA. Drugs with anticholinergic effects and cognitive impairment, falls and all-cause mortality in older adults: a systematic review and meta-analysis. Br J Clin Pharmacol. 2015;80(2):209-20.

4. Gorup E, Rifel J, Petek SM. Anticholinergic burden and most common anticholinergic-acting medicines in older general practice patients. Zdr Varst. 2018;57(3):140-7.

5. Salahudeen MS, Duffull SB, Nishtala PS. Anticholinergic burden quantified by anticholinergic risk scales and adverse outcomes in older people: a systematic review. BMC Geriatr. 2015;15:31.

6. Salahudeen MS, Nishtala PS. Examination and estimation of anticholinergic burden: current trends and implications for future research. Drugs Aging. 2016;33(5):305-13.

7. Coupland CAC, Hill T, Dening T, Morriss R, Moore M, Hippisley-Cox J. Anticholinergic drug exposure and the risk of dementia: a nested case-control study. JAMA Intern Med. 2019;179(8):1084-93.
8. Gray SL, Anderson ML, Dublin S, Hanlon JT, Hubbard R, Walker R, et al. Cumulative use of strong anticholinergics and incident dementia: a prospective cohort study. JAMA Intern Med. 2015;175(3):401-7.

9. Yeowell G, Smith P, Nazir J, Hakimi Z, Siddiqui E, Fatoye F. Real-world persistence and adherence to oral antimuscarinics and mirabegron in patients with overactive bladder (OAB): a systematic literature review. BMJ Open. 2018;8(11):e021889.

10. Maman K, Aballea S, Nazir J, Desroziers K, Neine M, Siddiqui E, et al. Comparative efficacy and safety of treatments for the management of overactive bladder: a systematic literature review and mixed treatment comparison. Value Health. 2013;16(3):A180.

11. Ivchenko A, Bodeker RH, Neumeister C, Wiedemann A. Anticholinergic burden and comorbidities in patients attending treatment with trospium chloride for overactive bladder in a real-life setting: results of a prospective non-interventional study. BMC Urol. 2018;18(1):80.

12. Szabo SM, Gooch K, Schermer C, Walker D, Lozano-Ortega G, Rogula B, et al. Association between cumulative anticholinergic burden and falls and fractures in patients with overactive bladder: US-based retrospective cohort study. BMJ Open. 2019;9(5):e026391.

13. Pfistermeister B, Tumena T, Gassmann KG, Maas R, Fromm MF. Anticholinergic burden and cognitive function in a large 
German cohort of hospitalized geriatric patients. PLoS ONE. 2017;12(2):e0171353.

14. Centre for Reviews and Dissemination, University of York. Systematic reviews: CRD's guidance for undertaking reviews in healthcare. York: Centre for Reviews and Dissemination, University of York; 2009.

15. Ades AE, Caldwel DM, Reken S, Welton NJ, Sutton AJ, Dias S. NICE DSU technical support document 7: evidence synthesis of treatment efficacy in decision making: a reviewer's checklist. 2012. https://www.nicedsu.org.uk/TSD7\%2520reviewer\%2520c hecklist.final.08.05.12.pdf. Accessed 12 Aug 2020.

16. Dias S, Welton NJ, Sutton AJ, Ades AE. NICE DSU technical support document 2: a generalised linear modelling framework for pairwise and network meta-analysis of randomised controlled trials. 2011.

17. Montedori A, Bonacini MI, Casazza G, Luchetta ML, Duca P, Cozzolino F, et al. Modified versus standard intention-to-treat reporting: are there differences in methodological quality, sponsorship, and findings in randomized trials? A cross-sectional study. Trials. 2011;12(1):58.

18. Higgins JPTGS. Cochrane handbook for systematic reviews of interventions. Version 5.1.0 [updated March 2011]. London: The Cochrane Collaboration; 2011.

19. Spiegelhalter D, Thomas A, Best N, Lunn D. OpenBUGS user manual. 2014. https://www.mrc-bsu.cam.ac.uk/bugs. Accessed 12 Aug 2020.

20. Abrams P, Gong Z, Creanga D. Fesoterodine versus tolterodine for overactive bladder in older and younger subjects. Urology. 2010;1:S42.

21. Capo JP, Lucente V, Forero-Schwanhaeuser S, He W. Efficacy and tolerability of solifenacin in patients aged $\geq 65$ years with overactive bladder: post-hoc analysis of 2 open-label studies. Postgrad Med. 2011;123(1):94-104.

22. Cardozo L, Castro-Diaz D, Gittelman M, Ridder A, Huang M. Reductions in overactive bladder-related incontinence from pooled analysis of phase III trials evaluating treatment with solifenacin. Int Urogynecol J Pelvic Floor Dysfunct. 2006;17(5):512-9.

23. Dubeau CE, Kraus SR, Griebling TL, Newman DK, Wyman JF, Johnson TM 2nd, et al. Effect of fesoterodine in vulnerable elderly subjects with urgency incontinence: a double-blind, placebo controlled trial. J Urol. 2014;191(2):395-404.

24. Hill S, Elhilali M, Millard RJ, Dwyer PL, Lheritier K, Kawakami FT, et al. Long-term darifenacin treatment for overactive bladder in patients aged 65 years and older: analysis of results from a 2-year, open-label extension study. Curr Med Res Opin. 2007;23(11):2697-704.

25. Sand PK, Heesakkers J, Kraus SR, Carlsson M, Guan Z, Berriman S. Long-term safety, tolerability and efficacy of fesoterodine in subjects with overactive bladder symptoms stratified by age: pooled analysis of two open-label extension studies. Drugs Aging. 2012;29(2):119-31.

26. Wagg A, Arumi D, Herschorn S, Angulo Cuesta J, Haab F, Ntanios F, et al. A pooled analysis of the efficacy of fesoterodine for the treatment of overactive bladder, and the relationship between safety, co-morbidity and polypharmacy in patients aged 65 years or older. Age Ageing. 2017;46(4):620-6.

27. Yokoyama O, Yamagami H, Hiro S, Hotta S, Yoshida M. Efficacy and safety of fesoterodine treatment for overactive bladder symptoms in elderly women with and without hypertension. Int J Urol. 2018;25(3):251-7.

28. Batista JE, Kolbl H, Herschorn S, Rechberger T, Cambronero J, Halaska M, et al. The efficacy and safety of mirabegron compared with solifenacin in overactive bladder patients dissatisfied with previous antimuscarinic treatment due to lack of efficacy: results of a noninferiority, randomized, phase IIIlb trial. Ther Adv Urol. 2015;7(4):167-79.
29. Cardozo L, Herschorn S, Snijder R, Siddiqui E, Chapple CR. Does BMI, gender or age affect efficacy/tolerability of solifenacin in the management of overactive bladder? Int Urogynecol J. 2017;28(3):477-88.

30. Chapple C, DuBeau C, Ebinger U, Rekeda L, Viegas A. Darifenacin treatment of patients $\geq 65$ years with overactive bladder: results of a randomized, controlled, 12-week trial. Curr Med Res Opin. 2007;23(10):2347-58.

31. Dubeau CE, Morrow JD, Kraus SR, Creanga D, Bavendam T. Efficacy and tolerability of fesoterodine versus tolterodine in older and younger subjects with overactive bladder: a post hoc, pooled analysis from two placebo-controlled trials. Neurourol Urodyn. 2012;31(8):1258-65.

32. Foote J, Glavind K, Kralidis G, Wyndaele JJ. Treatment of overactive bladder in the older patient: pooled analysis of three phase III studies of darifenacin, an M3 selective receptor antagonist. Eur Urol. 2005;48(3):471-7.

33. Frenkl T, Railkar RA, Shore N, Bailen J, Sutherland SE, Burke J, et al. A randomized, placebo controlled, crossover study to evaluate the effects of tolterodine on urodynamic parameters in patients with overactive bladder. Neurourol Urodyn. 2010;29(2):295-6.

34. Griebling TL, Kraus SR, Richter HE, Glasser DB, Carlsson M. Tolterodine extended release is well tolerated in older subjects. Int J Clin Pract. 2009;63(8):1198-204.

35. Kosilov K, Loparev S, Ivanovskaya M, Kosilova L. A randomized, controlled trial of effectiveness and safety of management of $\mathrm{OAB}$ symptoms in elderly men and women with standard-dosed combination of solifenacin and mirabegron. Arch Gerontol Geriatr. 2015;61(2):212-6.

36. Kraus SR, Ruiz-Cerd JL, Martire D, Wang JT, Wagg AS. Efficacy and tolerability of fesoterodine in older and younger subjects with overactive bladder. Urology. 2010;76(6):1350-7.

37. Lackner TE, Wyman JF, McCarthy TC, Monigold M, Davey C. Randomized, placebo-controlled trial of the cognitive effect, safety, and tolerability of oral extended-release oxybutynin in cognitively impaired nursing home residents with urge urinary incontinence. J Am Geriatr Soc. 2008;56(5):862-70.

38. Sand PK, Miklos J, Ritter H, Appell R. A comparison of extendedrelease oxybutynin and tolterodine for treatment of overactive bladder in women. Int Urogynecol J. 2004;15(4):243-8.

39. Sand PK, Johnson Ii TM, Rovner ES, Ellsworth PI, Oefelein MG, Staskin DR. Trospium chloride once-daily extended release is efficacious and tolerated in elderly subjects (aged $\geq 75$ years) with overactive bladder syndrome. BJU Int. 2011;107(4):612-20.

40. Wagg A, Wyndaele JJ, Sieber P. Efficacy and tolerability of solifenacin in elderly subjects with overactive bladder syndrome: a pooled analysis. Am J Geriatr Pharmacother. 2006;4(1):14-24.

41. Wagg A, Khullar V, Marschall-Kehrel D, Michel MC, Oelke M, Darekar A, et al. Flexible-dose fesoterodine in elderly adults with overactive bladder: results of the randomized, double-blind, placebo-controlled study of fesoterodine in an aging population trial. J Am Geriatr Soc. 2013;61(2):185-93.

42. Wagg A, Nitti V, Kelleher C, Auerbach S, Blauwet MB, Siddiqui E. Effects of the beta3-adrenoceptor agonist, mirabegron, on quality of life in older patients with overactive bladder: a post-hoc analysis of pooled data from 3 randomised phase 3 trials. J Urol. 2014;1:e338-e33939.

43. Wagg A, Cardozo L, Nitti VW, Castro-Diaz D, Auerbach S, Blauwet MB, et al. The efficacy and tolerability of the beta3-adrenoceptor agonist mirabegron for the treatment of symptoms of overactive bladder in older patients. Age Ageing. 2014;43(5):666-75.

44. Wagg A, Staskin D, Engel E, Herschorn S, Kristy RM, Schermer CR. Efficacy, safety, and tolerability of mirabegron in patients aged $\geq 65 \mathrm{yr}$ with overactive bladder wet: a phase IV, doubleblind, randomised, placebo-controlled study (PILLAR). Eur Urol. 2020;77(2):211-20. 
45. Zinner NR, Mattiasson A, Stanton SL. Efficacy, safety, and tolerability of extended-release once-daily tolterodine treatment for overactive bladder in older versus younger patients. J Am Geriatr Soc. 2002;50(5):799-807.

46. Malone-Lee JG, Bernard Walsh J, Maugourd MF. Tolterodine: a safe and effective treatment for older patients with overactive bladder. J Am Geriatr Soc. 2001;49(6):700-5.

47. Maman K, Aballea S, Nazir J, Desroziers K, Neine ME, Siddiqui $\mathrm{E}$, et al. Comparative efficacy and safety of medical treatments for the management of overactive bladder: a systematic literature review and mixed treatment comparison. Eur Urol. 2014;65(4):755-65.

48. Drake MJ, Nitti VW, Ginsberg DA, Brucker BM, Hepp Z, McCool $\mathrm{R}$, et al. Comparative assessment of the efficacy of onabotulinumtoxinA and oral therapies (anticholinergics and mirabegron) for overactive bladder: a systematic review and network metaanalysis. BJU Int. 2017;120(5):611-22.
49. Fest J, Pfalzgraf D, Weiss C, Hetjens S. Evaluating the efficacy and tolerability of mirabegron, a $\beta 3$-adrenoceptor agonist, for the treatment of overactive bladder: systematic review and network meta-analysis. J Clin Urol. 2017;10(6):557-67.

50. Kelleher C, Hakimi Z, Zur R, Siddiqui E, Maman K, Aballea S, et al. Efficacy and tolerability of mirabegron compared with antimuscarinic monotherapy or combination therapies for overactive bladder: a systematic review and network meta-analysis. Eur Urol. 2018;74(3):324-33.

51. Stijnen T, Hamza TH, Ozdemir P. Random effects meta-analysis of event outcome in the framework of the generalized linear mixed model with applications in sparse data. Stat Med. 2010;29(29):3046-67.

52. Sand PK, Johnson TM Jr, Rovner ES, Ellsworth PI, Oefelein MG, Staskin DR. Trospium chloride once-daily extended release is efficacious and tolerated in elderly subjects (aged $\geq 75$ years) with overactive bladder syndrome. J Urol. 2011;186(3):991-2.

\section{Affiliations}

\section{Greta Lozano-Ortega ${ }^{1}\left(\mathbb{D} \cdot\right.$ David R. Walker $^{2}{ }^{-} \cdot$ Karissa Johnston $^{1}\left(\mathbb{D} \cdot\right.$ Alexis Mickle $^{1} \cdot$ Sean Harrigan ${ }^{1}$. Basia Rogula ${ }^{1}$ (D) Rita M. Kristy ${ }^{2} \cdot$ John C. Hairston ${ }^{2} \cdot$ Carol R. Schermer $^{2}$}

Greta Lozano-Ortega

glozano-ortega@broadstreetheor.com

David R. Walker

david.walker@astellas.com

Karissa Johnston

kjohnston@broadstreetheor.com

Alexis Mickle

amickle@broadstreetheor.com

Sean Harrigan

sharrigan@broadstreetheor.com

Basia Rogula

brogula@broadstreetheor.com
Rita M. Kristy

rita.kristy@astellas.com

John C. Hairston

john.hairston@astellas.com

Carol R. Schermer

carol.schermer@astellas.com

1 Broadstreet Health Economics and Outcomes Research, Vancouver, BC, Canada

2 Astellas Pharma Global Development, Inc., Northbrook, IL, USA 